\title{
GEODESICS AND DEFORMED SPHERES
}

\author{
ABBAS M. FARIDI AND E. L. SCHUCKING
}

\begin{abstract}
A simple class of deformed 2-spheres is given whose intrinsic geometry and geodesics are described by elementary functions. Simple, explicit conditions for closed geodesics are obtained.
\end{abstract}

1. Introduction. Ellipsoids of revolution can be thought of as elementary examples of deformed spheres. However, while ellipsoids are extrinsically described by elementary functions, their intrinsic description uses elliptic functions. Thus, the study of geodesics on ellipsoids makes it necessary to employ elliptic functions. Since we are dealing here with one of the simplest examples for the global study of dynamical systems, one might like to study cases of deformed spheres where the geodesics and the intrinsic geometry are given in terms of elementary functions. We found such surfaces during a study of metrics which allow certain types of integrals which will be reported elsewhere.

2. The $\lambda$-spheres. We introduce a " $\lambda$-sphere" by means of the line element

$$
d s^{2}=d \theta^{2}+\frac{\sin ^{2} \theta}{1-\lambda \sin ^{2} \theta} d \varphi^{2},
$$

where $\theta$ ranges from 0 to $\pi$ and $\varphi$ is taken modulo $2 \pi$.

The metric is clearly regular at the north and south pole given by $\theta=0$ and $\theta=\pi$. The deformation parameter $\lambda$ is taken to be real and restricted to

$$
\lambda<\frac{1}{3} \text {. }
$$

For $\lambda=0$ a $\lambda$-sphere is metrically the unit sphere. A $\lambda$-sphere is a surface of revolution whose meridians $\varphi=$ const have length $\pi$. The $\lambda$-sphere is also symmetric with respect to reflection on the equator $\theta=\pi / 2$ given by

$$
\theta \rightarrow \pi-\theta, \quad \varphi \rightarrow \varphi
$$

and with respect to reflection in each meridian $\varphi_{0}$

$$
\varphi \rightarrow 2 \varphi_{0}-\varphi, \quad \theta \rightarrow \theta .
$$

A combination of the last two symmetries gives the result that the $\lambda$-sphere is symmetric under inversion with respect to each point on the equator. The equator of a $\lambda$-sphere has length $2 \pi(1-\lambda)^{-1 / 2}$. For a positive deformation parameter this leads to an oblate figure. For negative values of the parameter $\lambda$-spheres are spindle-like.

Received by the editors April 25, 1986.

1980 Mathematics Subject Classification (1985 Revision). Primary 53A05, 53C22. 
The area $A$ of a $\lambda$-sphere is obtained from

$$
\begin{aligned}
A & =2 \pi \int_{0}^{\pi}\left(1-\lambda \sin ^{2} \theta\right)^{-1 / 2} \sin \theta d \theta \\
& =4 \pi \times\left\{\begin{array}{ll}
\lambda^{-1 / 2} \operatorname{sh}^{-1}[\lambda /(1-\lambda)]^{1 / 2}, & \lambda>0 \\
1, & \lambda=0 \\
(-\lambda)^{-1 / 2} \sin ^{-1}[-\lambda /(1-\lambda)]^{1 / 2}, & \lambda<0
\end{array}\right\} .
\end{aligned}
$$

This gives for small $\lambda$ the area

$$
A \approx 4 \pi(1+\lambda / 2) .
$$

For $\lambda \rightarrow-\infty$ the area of a $\lambda$-sphere becomes arbitrarily small. This means that the shape approaches that of an infinitely thin spindle. The Gaussian curvature $K$ of a $\lambda$-sphere is given by

$$
K=\frac{1-3 \lambda+2 \lambda \sin ^{2} \theta}{\left(1-\lambda \sin ^{2} \theta\right)^{2}} .
$$

For $\lambda<\frac{1}{3}$ this curvature will always be positive. The curvature at the poles is given by $1-3 \lambda$ and at the equator by $(1-\lambda)^{-1}$. For $\lambda=\frac{1}{3}$ the poles become flat points. Since we want to restrict ourselves here to the positive curvature case we did impose the condition (2.2) on the deformation parameter.

Geodesics. A pleasant feature of the $\lambda$-spheres is that the equations of their geodesics can be completely integrated in terms of elementary functions. If a dot indicates differentiation with respect to arc-length $s$ the geodesics $\theta(s), \varphi(s)$ are derived from the integral

$$
\dot{\theta}^{2}+\frac{\sin ^{2} \theta}{1-\lambda \sin ^{2} \theta} \dot{\varphi}^{2}=1 .
$$

Rotational symmetry gives rise to the integral

$$
\frac{\sin ^{2} \theta}{1-\lambda \sin ^{2} \theta} \dot{\varphi}=\alpha=\text { const } .
$$

Instead of the energy integral (3.1) we can also employ the equation

$$
\ddot{\theta}=\frac{\sin \theta \cos \theta}{\left(1-\lambda \sin ^{2} \theta\right)^{2}} \dot{\varphi}^{2} .
$$

There are three types of geodesics on $\lambda$-spheres. The first kind are the meridians. For them the constant $\alpha$ vanishes since their longitude $\varphi$ is constant. They are closed and run through both poles.

Only one geodesic of the second kind exists which runs at constant $\theta$. We see from equation (3.3) that $\theta$ must be equal to $\pi / 2$. This geodesic is also closed and forms the equator.

The geodesics of the third kind are intermediate between meridians and equator. They will reach a smallest northern colatitude $\theta_{0}>0$ where $\dot{\theta}$ vanishes. At that point we have from (3.1)

$$
\frac{\sin ^{2} \theta_{0}}{1-\lambda \sin ^{2} \theta_{0}} \dot{\varphi}^{2}=1
$$


We obtain then from (3.2) that the integral $\alpha$ takes the value

$$
\alpha=\frac{\sin \theta_{0}}{\left(1-\lambda \sin ^{2} \theta_{0}\right)^{1 / 2}}>0
$$

The positive sign obtains if the arc-length $s$ of the geodesic is reckoned in the direction of increasing polar angle $\varphi$. We have

$$
0<\theta_{0}<\pi / 2 \text {. }
$$

For $\theta_{0}=0$ we obtain the meridians and for $\theta_{0}=\pi / 2$ the equator. The colatitude $\theta$ increases from its minimum $\theta_{0}$ till it reaches its maximum

$$
\theta_{1}=\pi-\theta_{0}
$$

where the geodesic attains a point closest to the south pole of the surface. Halfway in longitude between these two points the geodesic intercepted the equator $\theta=\pi / 2$. It now bends back northwards and will reach again the colatitude circle $\theta=\theta_{0}$, generally at a different value of $\varphi$ modulo $2 \pi$ and oscillate forever between the two colatitudes $\theta_{0}$ and $\theta_{1}$, which are symmetric to the equator. For a given value of $\lambda$ we may expect that there are certain values of the constant $\theta_{0}$ such that the geodesics close after having gone around the $\lambda$-sphere a number of times.

We restrict ourselves now to geodesics of the third kind and write instead of

$$
\frac{\sin ^{2} \theta}{1-\lambda \sin ^{2} \theta} \dot{\varphi}=\frac{\sin \theta_{0}}{\left(1-\lambda \sin ^{2} \theta_{0}\right)^{1 / 2}}=\alpha, \quad 0<\theta_{0}<\frac{\pi}{2} .
$$

Instead of arc-length $s$ we introduce the modified arc-length $\sigma$ defined by

$$
\sigma=s /\left(1-\lambda \sin ^{2} \theta_{0}\right)^{1 / 2}
$$

as parameter. One obtains then from (3.1), (3.8), and (3.9)

$$
\cos \theta=\cos \theta_{0} \cos \sigma
$$

where the zero-point for $\sigma$ was chosen such that

$$
\sigma=0: \theta=\theta_{0} \text {. }
$$

For $\sigma=\pi / 2$ the geodesic intersects the equator; for $\sigma=\pi$ it reaches a point furthest to the South at $\theta_{1}=\pi-\theta_{0}$ and returns for $\sigma=2 \pi$ back to the circle $\theta=\theta_{0}$. One obtains then from equations (3.8) and (3.9) that

$$
\begin{aligned}
\varphi-\varphi_{0} & =\int \frac{\sin \theta_{0} d \sigma}{1-\cos ^{2} \theta_{0} \cos ^{2} \sigma}-\sigma \lambda \sin \theta_{0} \\
& =\tan ^{-1}\left(\frac{\tan \sigma}{\sin \theta_{0}}\right)-\sigma \theta \sin \theta_{0} .
\end{aligned}
$$

The integration constant has been chosen such that the geodesic starts from the point $\left(\theta_{0}, \varphi_{0}\right)$ orthogonal to the meridian. It follows from (3.10) and (3.12) that the geodesic upon first return to the colatitude $\theta_{0}$ will have advanced in longitude by the amount $\Delta \varphi$,

$$
\Delta \varphi=2 \pi\left(1-\lambda \sin \theta_{0}\right)
$$

If

$$
1-\lambda \sin \theta_{0}=m / n,
$$

where $m$ and $n$ are integers without a common nontrivial divisor, the geodesic will close after $n$ oscillations which have wound $m$-times around the $\lambda$-sphere. 
4. Final remarks. For $\lambda<\frac{1}{3}$ the $\lambda$-spheres can be imbedded as surfaces of revolution in the 3-dimensional Euclidean space. One can take the contour

$$
x=\sin \theta\left(1-\lambda \sin ^{2} \theta\right)^{-1 / 2}, \quad z=\int\left[1-\cos ^{2} \theta /\left(1-\lambda \sin ^{2} \theta\right)^{3}\right]^{1 / 2} d \theta
$$

in the $x-z$ plane and rotate it about the $z$-axis. This results in a $\lambda$-sphere. The evaluation of the integral leads to a combination of elliptic integrals of all three kinds and does not appear to be helpful in elucidating the geometry. In this respect the $\lambda$-spheres are antithetical to the ellipsoids of revolution. It might be worthwhile checking whether the geoid is better approximated by a $\lambda$-sphere than by an ellipsoid of rotation.

Department of Physics, University of California, Santa Barbara, CaliforNIA 93106

Department of Physics, New York University, 4 Washington Place, New YORK, NEW YORK 10003 